DOI: https://dx.doi.org/10.26808/rs.ca.i8v1.01

International Journal of Computer Application (2250-1797)

Issue 8 Volume 1, January- February 2018

\title{
Estimation of Air Quality Index by Merging Neural Network with Principal Component Analysis
}

\section{Eman Sarwat and Ghada I. El-Shanshoury}

Radiation Safety Department, Nuclear and Radiological Regulatory Authority (NRRA)

\begin{abstract}
The objective of this study is to merge the Principal Component Analysis (PCA) with Artificial Neural Network (ANN) techniques to get the precise results in estimating the Air Quality Index (AQI). This study is applied for Ain Sokhna city for year 2014. Principal components score (PCs) of 10 historical air quality and meteorological parameters are generated and selected appropriately as the input variables in ANN models for predicting AQI. ANN and Multiple Linear Regression (MLR) models are applied and compared for four methods to estimate the AQI. Ten air pollutant and metrological parameters (actual data) are used as input data in ANN and MLR for creating the first method. The other three methods (Varimax, Equamax and Quartimax) are generated by rotating the actual data using PCA to reduce the ten parameters into four PCs for each PCA method. Consequently, the four PCs of each method are used as input data in ANN (PC-ANN) and MLR (PCR) models. The performance of the methods has been compared using Coefficient of Determination $\left(\mathrm{R}^{2}\right)$, Root Mean Square Error (RMSE), Mean Absolute Percentage Error (MAPE) and Mean Absolute Error (MAE). Considering the complexity of the air pollution phenomenon, it is found that the ANN model gives the best solution for all rotating PCs using Varimax method. The results show that the estimation of AQI by merging ANN with PCA (PC-ANN) gives more accurate results than merging MLR with PCA (PCR). This indicates that the ANN method can be applied successfully as a tool for decision making and problem solving for better atmospheric management.
\end{abstract}

Keywords: Air Quality Index (AQI), Principal Component Analysis (PCA), Artificial Neural Network (ANN).

\section{INTRODUCTION}

The examination of the locative characteristics in the design of air quality monitoring sites, distinguish the most specific parameters contributing to air pollution and predict the level of Air Quality Index (AQI) is required for environmental studies.

Air pollution is a mixture of many different substances and the exact contents vary depending on what sources of pollution are close by, area, the time of year and indeed the weather. Sources of air pollutant can be man-made, such as vapor from vehicles and smoke from burning fuels. While others, such as North African desert dust and radon gas, are natural. Air pollution is often separated into outdoor and indoor air pollution. In spite of the fact that both indoor and outdoor air pollutants have been shown to increment the hazard of cancer, it's imperative to keep the hazard in perspective [1].

Air pollution is a well-known environmental issue related with civilized zones around the world. Different monitoring software engineers have been undertaken to know the quality of air by creating vast amount of information on concentration of each air pollutant (e.g., $\mathrm{PM}_{10}, \mathrm{CO}, \mathrm{NO}_{\mathrm{x}}, \mathrm{SO}_{2}$, etc.) in different parts of the world. The huge information often do not pass on the air quality status to the scientific community, government authorities, policy makers, and in specific to the general public in a simple and direct way. This problem is addressed by defining the Air Quality Index (AQI) of a given area. AQI system plays a vital part in conveying to both decision-makers and the public the situation of ambient air quality that extends from good to dangerous [2]. AQI, which is too known as Air Pollution Index ( (API) has been developed and spread by numerous agencies in U.S. Canada, Europe, Australia, China, Indonesia, Taiwan, etc [3]. 
DOI: https://dx.doi.org/10.26808/rs.ca.i8v1.01

The initial iteration of the AQI standardized ambient pollutant concentrations to yield individual pollutant indices is utilized. This demonstrates were then weighted and summed to shape a single total air quality index. The AQI is an index determined by calculating the degree of contamination in the city or at the observing point [4].

This work aimed to find accurate manner for predicting the AQI that is used as a tool for effective dissemination of air quality information to people.

Air pollutant index (API) has been used as an indicator of air quality in air. In this paper, six criteria of air pollutants is studied as air pollutant index calculation that consists of; Carbon Monoxide $\left(\mathrm{CO}, \mu \mathrm{g} / \mathrm{m}^{3}\right)$, Nitric Oxide $\left(\mathrm{NO}, \mu \mathrm{g} / \mathrm{m}^{3}\right)$ Nitrogen Dioxide $\left(\mathrm{NO}_{2}, \mu \mathrm{g} / \mathrm{m}^{3}\right)$, Nitrogen Oxide $\left(\mathrm{NO}_{\mathrm{X}}, \mu \mathrm{g} / \mathrm{m}^{3}\right)$ Particulate Matter under 10 microns $\left(\mathrm{PM}_{10}, \mu \mathrm{g} / \mathrm{m}^{3}\right)$ and Sulphur Dioxide $\left(\mathrm{SO}_{2}, \mu \mathrm{g} / \mathrm{m}^{3}\right)$. As well, the study involved four meteorological parameters which are: Wind Direction (WD, deg), Wind Speed (WS, m/s), Ambient Temperature (AT, deg C) and Sigma Theta (SGT, deg). Computation of the AQI requires an air pollutant concentration over a specified averaging period, obtained from an air monitor or model. The radiationmonitoring network provides the air pollutant data for Ain Sokhna in year 2014.

The nearest neighbour method is applied to estimate missing records values of datasets. AQI is calculated according to Environmental Protection Agency (EPA) method [5].

PCA is used for classifying the predictor variables according to their interrelation, and to obtain parsimonious prediction model (i.e., model that depends on as few variables as necessary).

The current research work evaluates the performance characteristics of Principal Component Artificial Neural Network (PC-ANN) models against the Principal Component Regression (PCR) models. As well as, ANN prediction results are compared with Multiple Linear Regression (MLR) prediction results for original data. Four different criteria have been used to evaluate the effectiveness of each network and its ability to make precise prediction. The work reports that the use of principal component analysis (PCA) before the process of training the neural network (PC-ANN) can further improve the efficiency of generated models.

Statistical Data is analyzed using IBM $^{\circledR}$ SPSS $^{\circledR}$ statistics version 23 and Minitab statistics computer programs.

\subsection{Study Area and Data Collection}

\section{MATERIALS AND METHODS}

This study is applied for Ain Sokhna city in year 2014. The Latitude and Longitude of Ain Sokhna are $29^{\circ}$ $36^{\prime} \mathrm{N}$ and $32^{\circ} 19^{\prime} \mathrm{E}$, respectively.

The air quality and meteorological data are gathered from January to December 2014. The Radiation Monitoring Network in Egypt provides the recorded data [6]. The six criteria of air pollutants and four meteorological parameters are selected to study the influence of AQI values and the sources of pollution. The air pollutants parameters are: Carbon Monoxide (CO), Nitric Oxide (NO) Nitrogen Dioxide $\left(\mathrm{NO}_{2}\right)$, Nitrogen Oxide $\left(\mathrm{NO}_{\mathrm{X}}\right)$ Particulate Matter under 10 microns $\left(\mathrm{PM}_{10}\right)$ and Sulphur Dioxide $\left(\mathrm{SO}_{2}\right)$. As well, the meteorological parameters include Wind Direction (WD), Wind Speed (WS), Ambient Temperature (AT) and Sigma Theta (SGT). For the statistical analysis, the hourly data are employed to form a daily average that comprises 3650 datasets, 10 parameters $\times 365$ observations, which represent the average daily measured data in year 2014.

The Yamartino method computes the average sine and the average cosine of wind direction [7]. These two quantities are used to compute the epsilon value (the sine of sigma theta). Sigma theta is then computed as the arcsine of epsilon, multiplied by an interpolation factor to correct for large values of sigma theta [8]. Over the time interval to be averaged across, $n$ measurements of wind direction $(\theta)$ will be made and two totals are accumulated without storage of the $n$ individual values. At the end of the interval the calculations are as follows: with the average values of $\sin \theta$ and $\cos \theta$ defined as:

$$
S=\frac{1}{n} \sum_{i=1}^{n} \sin \theta_{i} \quad \mathrm{C}=\frac{1}{n} \sum_{i=1}^{n} \cos \theta_{i} \quad \varepsilon=\sqrt{1-\left(S^{2}+C^{2}\right)} \quad \sigma_{\theta}=\arcsin (\varepsilon)\left[1+\left(\frac{2}{\sqrt{3}}-1\right) \varepsilon^{3}\right]
$$


DOI: https://dx.doi.org/10.26808/rs.ca.i8v1.01

International Journal of Computer Application (2250-1797)

Issue 8 Volume 1, January- February 2018

\subsection{Statistical Techniques}

\subsubsection{Data Pre-Treatment}

In this study, the prediction model is developed using 3650 datasets. Six criteria of air pollutants and four meteorological parameters are employed in this study. The air pollutants parameters are mention in the previous section. Furthermore, the nearest neighbour method is applied using IBM $^{\circledR}$ SPSS $^{\circledR}$ statistics version 23 to estimate missing values. The missing data percentage recorded about $3 \%$ of the original data sets suggesting that nearest neighbor method can provide a simple scheme in which the endpoint of the gaps is used as estimates for all missing values. The equation for the nearest neighbour method is given by:

$$
y=y_{1} \text { if } x \leq x_{1}+\frac{x_{2}-x_{1}}{2} \quad \text { or } \quad y=y_{2} \text { if } x>x_{1}+\frac{x_{2}-x_{1}}{2}
$$

where $y$ is the interpolant, $x$ is the time point of the interpolant, $y_{1}$ and $x_{1}$ are the coordinates of the starting point of the gap, and $y_{2}$ and $x_{2}$ are the endpoints of the gap $[9,10]$.

\subsubsection{AQI by Using EPA Method (EPAQI)}

The AQI measures daily pollution index of the pollutants for which EPA has established National Ambient Air Quality Standards (NAAQS). The index combines the NAAQS with an epidemiological function to determine a descriptor of human health effects due to short-term exposure (24 hour or less) to each pollutant. The index for a pollutant is calculated using the mathematical expression [5,11]

$$
I_{P}=\frac{I_{\text {high }}-I_{\text {low }}}{B P_{\text {high }}-B P_{\text {low }}}\left(C_{p}-B P_{\text {low }}\right)+I_{\text {low }}
$$

where,

$I_{P}$ is the index value for pollutant $(P), C_{p}$ is the truncated concentration of pollutant $P, B P_{\text {low }}$ is the concentration breakpoint that is $\leq C_{p}, B P_{\text {high }}$ is the concentration breakpoint that is $\geq C_{p}, I_{\text {low }}$ is the index breakpoint corresponding to $B P_{\text {low }}$ and $I_{\text {high }}$ is the index breakpoint corresponding to $B P_{\text {high }}$.

The indexes for each of the pollutants $\mathrm{CO}(\mathrm{ppm}), \mathrm{NO}_{2}(\mathrm{ppb}), \mathrm{SO}_{2}(\mathrm{ppb})$, and $\mathrm{PM}_{10}\left(\mu \mathrm{g} / \mathrm{m}^{3}\right)$ are obtained from Eq. 2 using their respective break points and associated AQI values [5]. Having calculated $I_{P}$ of each pollutant, the EPAQI is evaluated by considering the maximum index value $\left(I_{P}\right)$ of the single pollutant. Mathematically, it is expressed as EPAQI $=\operatorname{Max}\left(I_{P}\right)[3]$.

\subsubsection{Multiple Linear Regressions (MLR)}

Multiple linear regression (MLR) is widely used in atmospheric modeling. This technique has been used to investigate the relationship among various independent and dependent variables by fitting a linear equation to observed data [12] and to give the percentage of the contribution of each parameter to the atmospheric pollution [13]. In this study, it is used to justify the relationship between the air quality with meteorological parameters (10 parameters) as independent variables and total AQI data as dependent variable. The model is obtained using equation (3):

$$
y_{i}=\beta_{0}+\beta_{1} x_{1}+\beta_{2} x_{2}+\ldots \ldots+\beta_{p-1} x_{p-1}+\varepsilon
$$

where,

$y_{i}$ is the response variable, $x_{1}, x_{2}, \ldots, x_{p-1}$ are the explanatory variable, $\beta_{0}, \beta_{1}, \beta_{2}, \ldots, \beta_{p-1}$ are regression coefficients and $\varepsilon$ is an error associated with the regression.

\subsubsection{Principal Component Analysis (PCA)}

In Factor analysis, the technique of Principal Component Analysis (PCA) is used. The basic purpose of PCA is to account for the total variation among the ' $n$ ' number of subjects (variables) in $p$-dimensional space by forming a new set of orthogonal and uncorrelated composite variates. Each member of the new set of variates is a linear combination of the original set of measurements. The linear combinations are generated in such a manner that each of the successive composite variates will account for a smaller portion of the total variation. The first composite (principal component) will have the largest variance; the second will have a variance 
DOI: https://dx.doi.org/10.26808/rs.ca.i8v1.01

International Journal of Computer Application (2250-1797)

Issue 8 Volume 1, January- February 2018

smaller than the first but larger than the third, and so on. If first few principal components (or, eigenvectoreigenvalue pairs) account for more than $60 \%$ of the total variance, then there is hardly any requirement in taking more principal components (PCs) to compute the composite (overall) Air Quality Index. The higher order PCs explain only minimal amounts of total variance and are, therefore, treated as noise [3].

Factor analysis has several rotation methods, such as varimax, equamax and quartimax. Varimax method is an orthogonal rotation method that minimizes the number of variables that have high loadings on each factor. This method simplifies the interpretation of the factors. Equamax method is a rotation method that is a combination of the Varimax method, which simplifies the factors, and the Quartimax method, which simplifies the variables. The number of variables that load highly on a factor and the number of factors needed to explain a variable are minimized. Quartimax method is a rotation method that minimizes the number of factors needed to explain each variable. This method simplifies the interpretation of the observed variables [14].

The PCs generated by PCA are sometimes not readily interpreted; therefore, it is advisable to rotate the PCs by Varimax rotation with Kaiser Normalization. Varimax rotation ensures that each variable is maximally correlated with only one PC and a near zero association with the other components [15]. However, the Equamax and Quartimax rotation methods with Kaiser Normalization is also applied. Only the PCs with eigenvalues more than 1 are considered significant in the rotations analysis. The PCs values which are greater than $0.75(>0.75)$ is considered as "strong", The values range from $0.50-0.75(0.50 \geq$ factor loading $\geq 0.75)$ is considered as "moderate", and the values range from $0.30-0.49(0.30 \geq$ factor loading $\geq 0.49)$ is considered as "weak" factor loadings [16].The PC coefficient with values greater than 0.5 is selected. The analysis of PCA was implemented using Minitab software. The PCs can expressed as Equation 4:

$$
Z_{i j}=a_{i 1} x_{1 j}+a_{i 2} x_{2 j}+a_{i 3} x_{3 j}+\ldots \ldots \ldots . . .+a_{i m} x_{m j}
$$

where $z$ is the component score, $a$ is the component loading, $x$ is the measured value of the variable, $i$ is the component number, $j$ is the sample number, and $m$ is the total number of variables [9].

\subsubsection{Principle Component Regression (PCR)}

Principal Component Regression (PCR) combines the PCA and OLS (ordinary least square method). In principal components PCR, principal components analysis (PCA) is used to decompose the independent $(x)$ variables into an orthogonal basis (the principal components), and select a subset of those components as the variables to predict $y$. The basic idea behind PCR is to calculate the principal components and then use some of these components as predictors in a linear regression model fitted using the typical least squares procedure. By using PCR, it can easily perform dimensionality reduction on a high dimensional dataset and then fit a linear regression model to a smaller set of variables, while at the same time keep most of the variability of the original predictors. PCR tends to perform well when the first principal components are enough to explain most of the variation in the predictors [17].

\subsection{Artificial Neural Networks (ANNs)}

Artificial neural networks (ANNs) have been successfully used for classification, prediction, and association in different problem domains. ANNs have the ability to approximate any nonlinear mathematical function, which is useful especially when the relationship between the variables is not known or is complex. The most common type of ANN was tested in this research - the multilayer perceptron (MLP), a feed forward network that can use various algorithms to minimize the objective function, such as backpropagation, conjugate gradient, and other. A simplified architecture of a MLP ANN is presented in Figure 1.

The input layer of an ANN consists of $\mathrm{n}$ input units with values $x_{i}, i=1,2, \ldots, \mathrm{n}$, and randomly determined initial weights $w_{i}$ usually from the interval [-1,1]. Each unit in the hidden (middle) layer receives the weighted sum of all $x_{i}$ values as the input. The output of the hidden layer denoted as $y_{c}$ is computed by summing the inputs multiplied with their weights, according to Equation 5 [18]: 
DOI: https://dx.doi.org/10.26808/rs.ca.i8v1.01

$$
y_{c}=f \sum_{i=1}^{n} w_{i} x_{i}
$$

where,

$f$ is the activation function selected by the user (sigmoid, tangent hyperbolic, exponential, linear, step or other).

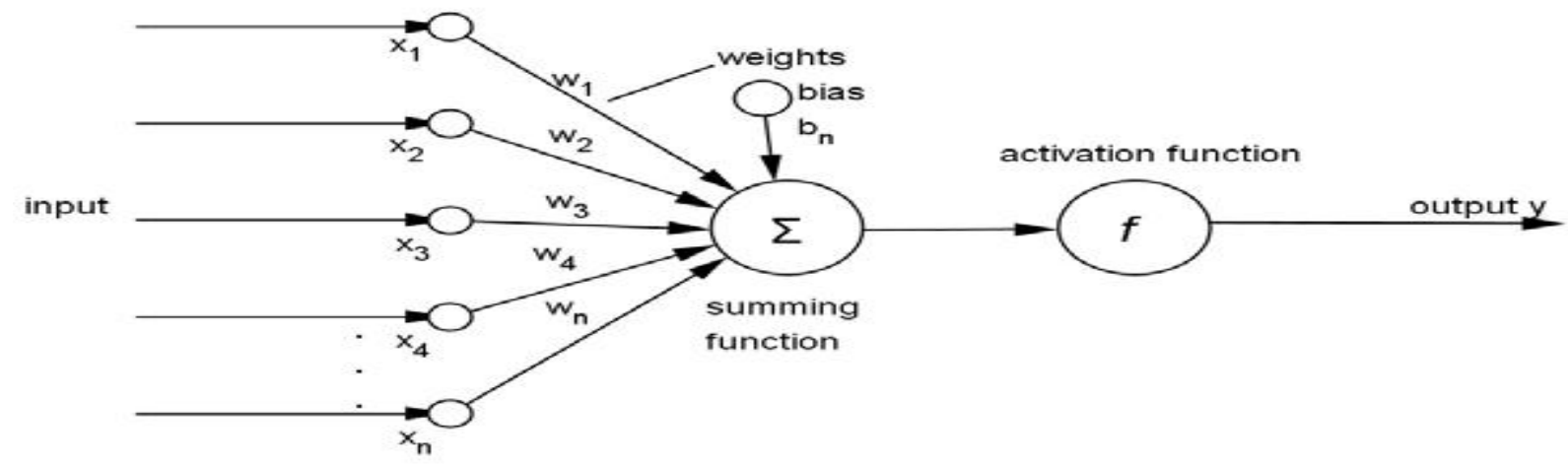

Fig. 1: Architecture of ANN

In this work, the back propagation (BP) ANN was used in the development of all the prediction models. The Activation Transfer Function of a back-propagation network is usually a differentiable Sigmoid (S-shape) function, which helps to apply the non-linear mapping from inputs to outputs [15]. A three layer backpropagation ANN is used in this study. The number of input and output neurons is determined by the nature of the problem under study. In this study, the networks are trained, tested and validated with one hidden layer and 3 to 6 neurons in the hidden layer. The output neuron (layer) gives the predicted AQI value which gives the best results.

\subsection{Merging PCA with Artificial Neural Networks}

The modeling designing is led by the idea to find the most efficient ANN model that will be able to classify students according to their entrepreneurial intentions with a satisfactory accuracy but with a minimal number of predictors needed as the input to the model. The aims are to lower the training time of the ANN, and to lower the time needed for the respondents to fill up the survey, which means to find the minimal number of items needed to predict the output variable. Therefore, PCA is performed to generate the principal components (PCs) and used as input variables in the AQI estimation model that occurs by using ANN approach (PC-ANN).

In this work, four different regression models are developed with different input variables

Method 1 is developed based on the original raw data (Ten parameters) using MLR and ANN.

Method 2 is developed based on the four PCs with Varimax rotation $\left(\right.$ Four PC $_{\mathrm{V}}$ ) using PCR and PC-ANN. Method 3 is developed based on the four PCs with Equamax rotation (Four $\mathrm{PC}_{\mathrm{E}}$ ) using PCR and PC-ANN. Method 4 is developed based on the four PCs with Quartimax rotation (Four $\mathrm{PC}_{\mathrm{Q}}$ ) using PCR and PC-ANN.

Trial-and-error procedure with one and three to six neutron hidden layers in the network structure is examined in order to reach the best model for estimation of AQI values.

\subsection{Determination of Model Performance}

Four different criteria have been used to evaluate the effectiveness and performance of ANN, MLR, PCANN and PCR models to make precise prediction. The evaluated criteria are: Coefficient of Determination $\left(R^{2}\right)$, Root Mean Square Error (RMSE), Mean Absolute Percentage Error (MAPE) and Mean Absolute Error 
DOI: https://dx.doi.org/10.26808/rs.ca.i8v1.01

(MAE) [19]. Here, lower value of RMSE, MAPE, MAE and higher value of $R^{2}$ give more accurate prediction.

$$
\mathrm{R}^{2}=1-\frac{\sum_{i=1}^{n}\left(\left(y_{i}-\hat{y_{i}}\right)^{2}\right.}{\sum_{i=1}^{n}\left(\left(y_{i}-\overline{y_{i}}\right)^{2}\right.}, \quad R M S E=\sqrt{\frac{\sum_{i=1}^{n}\left(\left(y_{i}-\hat{y}_{i}\right)^{2}\right.}{n}}, \quad M A P E=\left(\frac{\sum_{i=1}^{n}\left|y_{i}-\hat{y}_{i}\right| / y_{i}}{n}\right) * 100, \quad M A E=\frac{\sum_{i=1}^{n}\left|y_{i}-\hat{y_{i}}\right|}{n}
$$

Where, $y_{i}$ is the observed values of AQI levels, $\hat{y}_{i}$ is the predicted values of AQI levels, $\bar{Y}$ is the mean value of AQI levels and $n$ is the total number of samples. Evaluation of the results is based on the lowest values of RMSE, MAPE, MAE and the highest value of $\mathrm{R}^{2}$.

The performance of ANN prediction results are compared with MLR prediction results for the first method. As well as, the performance of PC-ANN prediction results are compared with PCR prediction results for methods 2-4. Then, the performance values of ANN models are compared with each other for all methods to obtain an appropriate method for predicting the AQI.

\section{RESULTS AND DISCUSSIONS}

\subsection{Principal Component Analysis}

The PCA obtained on the covariance matrix revealed that 10 components with eigenvalue greater than 1 can be produced from the data. Eigenvalues are most commonly reported in factor analysis. They are calculated and used in deciding how many factors to extract in the overall factor analysis. The factor with the largest eigenvalue has the most variance and so on, down to factors with small or negative eigenvalues that are usually omitted from solutions.

The scree plot of eigenvalues obtained from the sample is presented in Figure 2.

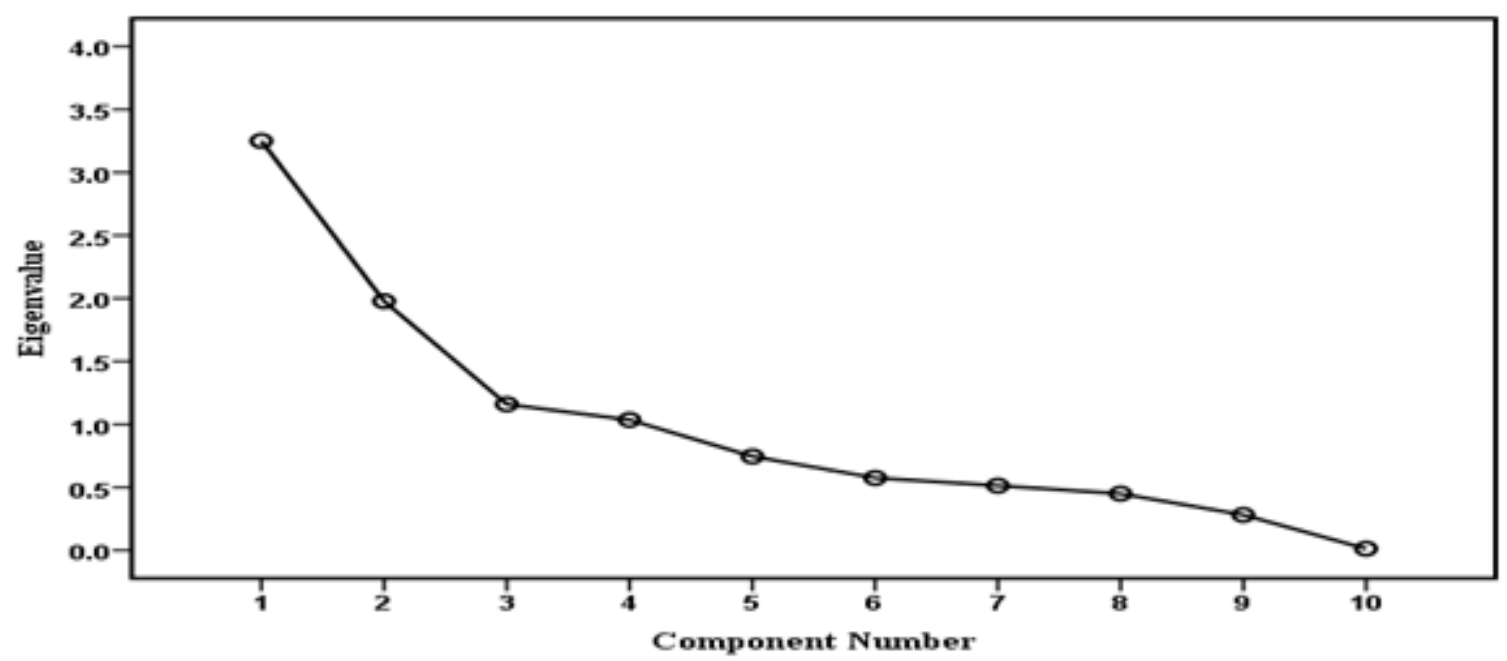

Fig. 2: Scree Plot of the Principal Component eigenvalues

From the scree plot Figure, out of the ten principal components (PCs) generated, only four PCs with eigenvalues greater than 1 are selected for inserting them in the feed-forward ANN and multiple linear regression as input variables. From PCA results, the selected parameters represent $74.2 \%$ of the total variance for all PCs rotation methods. The PCs coefficients with values greater than 0.5 are selected.

The results of the four PCs from the loading of PCA using Varimax Rotation are given in Table 1. Ten variables with moderate and strong loadings (noted in bold) are included in four selected RPCs. The Table 
DOI: https://dx.doi.org/10.26808/rs.ca.i8v1.01 International Journal of Computer Application (2250-1797) Issue 8 Volume 1, January- February 2018

shows that the first principal component explains the highest percentage of total variance (29.6\%), while the second rotated PC accounts for $22.0 \%$ of the total variance, the third component explains around $11.4 \%$ of total variance and the forth PC accounts for $11.2 \%$ of the total variance. Table 1 shows that the parameters consist in each PC may be positive or negative sign. The first PC explains the positive relationship amongst the four pollution data $\left(\mathrm{NO}_{\mathrm{x}}, \mathrm{NO}_{2}, \mathrm{NO}\right.$ and $\mathrm{SO}_{2}$ ). The PC2 (Factor 2) identifies the negative loading of wind direction and positive high loading of sigma theta and ambient temperature. In PC2, wind direction has a negative relationship with sigma theta and ambient temperature. PC3 identifies the high positive loading of carbon monoxide (CO). PC4 is accounted by positive contributions of $\mathrm{PM}_{10}$ and WS

Table 1: Rotated Factor Loadings and Communalities, Varimax Rotation

\begin{tabular}{|c|c|c|c|c|c|}
\hline Variable & PC1 & PC2 & PC3 & PC4 & Communality \\
\hline $\mathrm{NO}_{\mathrm{x}}$ & $\mathbf{0 . 9 2 4}$ & -0.299 & -0.018 & 0.032 & 0.945 \\
$\mathrm{NO}_{2}$ & $\mathbf{0 . 8 6 0}$ & -0.377 & -0.067 & -0.017 & 0.887 \\
$\mathrm{NO}$ & $\mathbf{0 . 7 8 0}$ & -0.008 & 0.103 & 0.145 & 0.640 \\
$\mathrm{SO}_{2}$ & $\mathbf{0 . 5 8 2}$ & 0.246 & 0.481 & -0.011 & 0.631 \\
$\mathrm{SGT}$ & 0.057 & $\mathbf{0 . 8 1 7}$ & -0.052 & -0.109 & 0.685 \\
$\mathrm{WD}$ & 0.150 & $\mathbf{- 0 . 7 3 9}$ & -0.094 & 0.012 & 0.577 \\
$\mathrm{ATM}$ & -0.326 & $\mathbf{0 . 7 2 5}$ & 0.124 & 0.175 & 0.678 \\
$\mathrm{CO}$ & 0.015 & 0.029 & $\mathbf{0 . 8 9 9}$ & -0.039 & 0.810 \\
$\mathrm{PM}$ & 0.190 & 0.069 & -0.096 & $\mathbf{0 . 8 9 5}$ & 0.852 \\
$\mathrm{WS}$ & -0.500 & -0.399 & 0.231 & $\mathbf{0 . 5 0 5}$ & 0.717 \\
\hline Eignvalue & 2.9598 & 2.1958 & 1.1438 & 1.1233 & 7.4225 \\
\% Var & 29.6 & 22.0 & 11.4 & 11.2 & 74.2 \\
\hline
\end{tabular}

Rotated factor loadings of Equamax Rotation are given in Table 2. The Table shows that the first PC explains the positive relationship amongst the four pollution data $\left(\mathrm{NO}_{\mathrm{x}}, \mathrm{NO}_{2}, \mathrm{NO}\right.$ and $\left.\mathrm{SO}_{2}\right)$ and the negative loading of wind speed with $29.4 \%$ of total variance. The PC2 also identifies the negative loading of wind direction and high positive loading of sigma theta and ambient temperature with $21.9 \%$ of total variance. PC 3 is composed of positive loading of $\mathrm{CO}$ and $\mathrm{SO}_{2}$ with $11.6 \%$ of total variance. $\mathrm{PC} 4$ is accounted by positive contributions of $\mathrm{PM}_{10}$ and WS with $11.3 \%$ of total variance

Table 2: Rotated Factor Loadings and Communalities, Equamax Rotation

\begin{tabular}{|c|c|c|c|c|c|}
\hline Variable & PC1 & PC2 & PC3 & PC4 & Communality \\
\hline $\mathrm{NO}_{\mathrm{x}}$ & $\mathbf{0 . 9 2 2}$ & -0.308 & 0.012 & 0.028 & 0.945 \\
$\mathrm{NO}_{2}$ & $\mathbf{0 . 8 5 8}$ & -0.385 & -0.041 & -0.020 & 0.887 \\
$\mathrm{NO}$ & $\mathbf{0 . 7 7 7}$ & -0.017 & 0.133 & 0.138 & 0.640 \\
$\mathrm{SO}_{2}$ & $\mathbf{0 . 5 6 5}$ & 0.228 & $\mathbf{0 . 5 1 0}$ & -0.018 & 0.631 \\
$\mathrm{SGT}$ & 0.066 & $\mathbf{0 . 8 1 5}$ & -0.030 & -0.126 & 0.685 \\
$\mathrm{WD}$ & 0.146 & $\mathbf{- 0 . 7 3 8}$ & -0.105 & 0.024 & 0.577 \\
$\mathrm{ATM}$ & -0.322 & $\mathbf{0 . 7 2 9}$ & 0.126 & 0.165 & 0.678 \\
$\mathrm{CO}$ & -0.022 & 0.007 & $\mathbf{0 . 8 9 9}$ & -0.032 & 0.810 \\
$\mathrm{PM}$ & 0.204 & 0.087 & -0.093 & $\mathbf{0 . 8 9 1}$ & 0.852 \\
$\mathrm{WS}$ & $\mathbf{- 0 . 5 0 7}$ & -0.388 & 0.197 & $\mathbf{0 . 5 2 0}$ & 0.717 \\
\hline Eignvalue & 2.9358 & 2.1939 & 1.1635 & 1.1293 & 7.4225 \\
\% Var & 29.4 & 21.9 & 11.6 & 11.3 & 74.2 \\
\hline
\end{tabular}

Rotated factor loadings of Quartimax Rotation are given in Table 3. It is obvious from Table 3 that the first PC illustrates there is around $29.8 \%$ of total variance explains positive relationship amongst the four 
DOI: https://dx.doi.org/10.26808/rs.ca.i8v1.01 International Journal of Computer Application (2250-1797)

Issue 8 Volume 1, January- February 2018

pollution data $\left(\mathrm{NO}_{\mathrm{x}}, \mathrm{NO}_{2}, \mathrm{NO}\right.$ and $\left.\mathrm{SO}_{2}\right)$. The $\mathrm{PC} 2$ also identifies the negative loading of wind direction and high positive loading of sigma theta and ambient temperature with $21.9 \%$ of total variance. PC3 is accounted by strong positive contributions of $\mathrm{CO}$ with $11.3 \%$ of total variance. PC4 is composed of strong positive loading of $\mathrm{PM}_{10}$ with $11.2 \%$ of total variance

Table 3: Rotated Factor Loadings and Communalities, Quartimax Rotation

\begin{tabular}{|c|c|c|c|c|c|}
\hline Variable & PC1 & PC2 & PC3 & PC4 & Communality \\
\hline $\mathrm{NO}_{\mathrm{x}}$ & $\mathbf{0 . 9 2 7}$ & -0.290 & -0.036 & 0.034 & 0.945 \\
$\mathrm{NO}_{2}$ & $\mathbf{0 . 8 6 2}$ & -0.369 & -0.081 & -0.017 & 0.887 \\
$\mathrm{NO}$ & $\mathbf{0 . 7 8 1}$ & 0.000 & 0.084 & 0.150 & 0.640 \\
$\mathrm{SO}_{2}$ & $\mathbf{0 . 5 9 2}$ & 0.262 & 0.461 & -0.006 & 0.631 \\
$\mathrm{SGT}$ & 0.048 & $\mathbf{0 . 8 1 7}$ & -0.071 & -0.097 & 0.685 \\
$\mathrm{WD}$ & 0.155 & $\mathbf{- 0 . 7 3 9}$ & -0.082 & 0.003 & 0.577 \\
$\mathrm{ATM}$ & -0.332 & $\mathbf{0 . 7 2 2}$ & 0.118 & 0.182 & 0.678 \\
$\mathrm{CO}$ & 0.037 & 0.048 & $\mathbf{0 . 8 9 7}$ & -0.044 & 0.810 \\
$\mathrm{PM}$ & 0.181 & 0.056 & -0.096 & $\mathbf{0 . 8 9 8}$ & 0.852 \\
$\mathrm{WS}$ & -0.493 & -0.406 & 0.255 & 0.494 & 0.717 \\
\hline Eignvalue & 2.9767 & 2.1946 & 1.1321 & 1.1192 & 7.4225 \\
\% Var & 29.8 & 21.9 & 11.3 & 11.2 & 74.2 \\
\hline
\end{tabular}

\subsection{Prediction of the AQI Using MLR and PCR Models}

In this study, MLR is used for predicting the AQI by justifying the relationship between the air quality with meteorological parameters (10 parameters) as independent variables and total AQI data as dependent variable. The first model is obtained using the following equation (Method 1):

$$
\begin{aligned}
A Q I= & 31.7+0.182 \mathrm{NO}_{\mathrm{X}}-0.819 \mathrm{NO}_{2}-0.830 \mathrm{NO}+0.208 \mathrm{SO}_{2}+0.16 \mathrm{CO}+0.795 \mathrm{PM}_{10}+0.382 \mathrm{SGT}+ \\
& 0.0368 \mathrm{WD}-0.734 \mathrm{ATM}-6.75 \mathrm{WS}
\end{aligned}
$$

PCR is calculated by a two-step method. First, a principal component analysis is carried out on the actual variables. The four generating PCs are then used as predictors in a MLR (using PCs that noted in bold).

PCR is carried out for each rotation method that mention above (Table 1-3) for methods 2-4. PCR can reveal the inherent linear structure of a dataset and thus reduce the dimension of predicting variables.

According to Method 2, the PCR equation for predicting AQI is calculated as follows:

$A Q I=0.03-0.0832 P C 1-0.0182 P C 2+0.16 P C 3+0.869 P C 4$

Where, PCs are deduced from Table $1\left(P C 1=0.924 N O x+0.860 \mathrm{NO}_{2}+0.780 \mathrm{NO}+0.582 \mathrm{SO}_{2}\right.$,

$\boldsymbol{P C 2}=0.817$ SGT $-0.739 \mathrm{WD}+0.725 \mathrm{ATM}, \boldsymbol{P C 3}=0.899 \mathrm{CO}$ and $\left.\boldsymbol{P C 4}=0.895 P M_{10}+0.505 \mathrm{WS}\right)$

Methods 3 and 4 are calculated as the same manner of method 2.

\subsection{Prediction of the AQI Using ANN and PC-ANN Models}

In Method 1; original raw parameters are used as inputs; the optimum neuron in the hidden layers is 8 neurons.

In Methods 2, 3 and 4; four principal component scores for each of Varimax, Equamax and Quartimax rotation method are used as inputs; the three layer network is used with four neurons in the input layer, 3-6 neurons in the hidden layer and one neuron in the output layer.

The different neural network for the four methods has been summarized in Table 4 . 
DOI: https://dx.doi.org/10.26808/rs.ca.i8v1.01 International Journal of Computer Application (2250-1797) Issue 8 Volume 1, January- February 2018

Table 4: Neural Network model structure for the four methods

\begin{tabular}{|c|c|c|c|c|}
\hline Network methods & $\begin{array}{c}\text { Number of } i / p \\
\text { neurons }\end{array}$ & Input neurons detail & $\begin{array}{c}o / p \text { neurons } \\
\text { detail }\end{array}$ & $\begin{array}{c}\text { Number of neurons } \\
\text { In hidden layers }\end{array}$ \\
\hline Method 1 & 10 & 10 actual parameters & AQI & 8 \\
Method 2 & 4 & 4 PCs using Varimax & AQI & 6 \\
Method 3 & 4 & 4 PCs using Equamax & AQI & 4 \\
Method 4 & 4 & 4 PCs using Quartimax & AQI & 3 \\
\hline
\end{tabular}

Table 4 illustrates the number of neurons of the hidden layer for the four methods. The number of hidden layer neurons change during the prediction stage until the minimum prediction error is reached.

\subsection{Comparative Performance of Linear Regression and Feed-Forward ANN Models for Methods 1-4}

Ten air pollutant and metrological parameters (original data) are used as input data in ANN and MLR for constructing the first method. The other methods are generated by rotating the original data using three PCA methods (Varimax, Equamax and Quartimax) to reduce the ten parameters into four PCs for each PCA method. Consequently, the four PCs of each method are used as input data in ANN and MLR models. The performance of the models has been compared using RMSE, MAPE, MAE and $\mathrm{R}^{2}$.

Comparison between ANN, MLR, PC-ANN and PCR models for the four methods is shown in Table 5.

Table 5: Comparison between ANN, MLR, PC-ANN and PCR models for the four methods

\begin{tabular}{|c|c|c|c|}
\hline & $\begin{array}{l}\text { Models } \\
\text { Methods } \\
\end{array}$ & ANN & MLR \\
\hline \multirow{5}{*}{ Actual parameters } & Method 1 & & \\
\hline & RMSE & 0.738138433 & 12.77368 \\
\hline & MAPE & 0.994643773 & 9.943526 \\
\hline & MAE & 0.470259485 & 7.215754 \\
\hline & $\mathrm{R}^{2}$ & 0.99986 & 0.9562 \\
\hline \multirow{6}{*}{ Varimax PCs } & & PC-ANN & PCR \\
\hline & Method 2 & & \\
\hline & RMSE & 0.664864365 & 13.86453 \\
\hline & MAPE & 0.909452377 & 10.75272 \\
\hline & MAE & 0.429028015 & 7.795231 \\
\hline & $\mathrm{R}^{2}$ & 0.9999 & 0.9484 \\
\hline \multirow{5}{*}{ Equamax PCs } & Method 3 & & \\
\hline & RMSE & 1.377942542 & 13.86384 \\
\hline & MAPE & 1.535189768 & 10.82454 \\
\hline & MAE & 0.80805378 & 7.82207 \\
\hline & $\mathrm{R}^{2}$ & 0.9995 & 0.9484 \\
\hline \multirow{5}{*}{ Quartimax PCs } & Method 4 & & \\
\hline & RMSE & 1.22929989 & 13.98869 \\
\hline & MAPE & 1.886871321 & 13.59725 \\
\hline & MAE & 0.992499266 & 8.888763 \\
\hline & $\mathrm{R}^{2}$ & 0.9995 & 0.9490 \\
\hline
\end{tabular}


DOI: https://dx.doi.org/10.26808/rs.ca.i8v1.01 International Journal of Computer Application (2250-1797)

Issue 8 Volume 1, January- February 2018

According to performance evaluative criteia it is obvious from Table 5 that the Varimax rotated PCs method is combined with ANN model (PC-ANN using Varimax method) and gives the best estimation results for AQI when compares with methods 1,3 and 4. As well as, method 1 shows that the ANN model with actual data followed method 2 in the accurate estimation results. Table 4 also shows that using ANN or PC-ANN model for all methods gives the best approach to estimate AQI than MLR or PCR models.

\subsection{The Relationship between Estimated and Actual AQI Using PC-ANN and PCR}

Three relation functions are drawn for the estimated AQI comparisons. Linear, Quadratic and polynomial $4^{\text {th }}$ degree are deduced. All relation functions of PC-ANN and actual data have the same line (identical linear line) and determination coefficients $\left(\mathrm{R}^{2} \approx 1\right)$ as shown in Fig. 3 . In contrast, the polynomial $4^{\text {th }}$ degree relation between PCR and actual data has the best relation between them with determination coefficients less than PC-ANN as shown in Fig. 4. Consequently, the linear relation between the estimated AQI of PC-ANN and actual data is considered the easier for calculating or getting to the actual data $(A Q I=0.9997 x+0.1768)$. In addition, it gives a high accurate resluts. Moreover, the polynomial $4^{\text {th }}$ degree relation between PCR and actual data is considered the best relation between them but less accuracy in calculating the actual data than any relations deduced in Fig. 3.

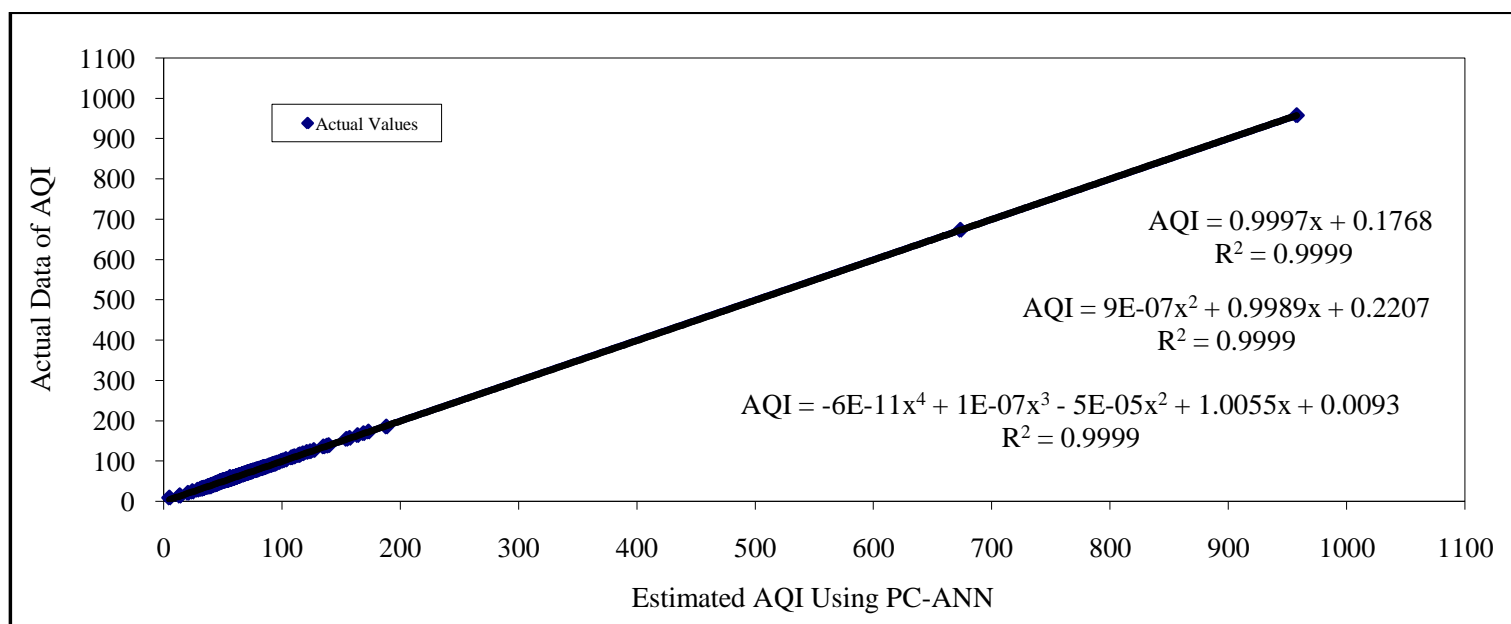

Fig. 3: Estimated AQI Using PC-ANN of Varimax Method Versus Actual Data

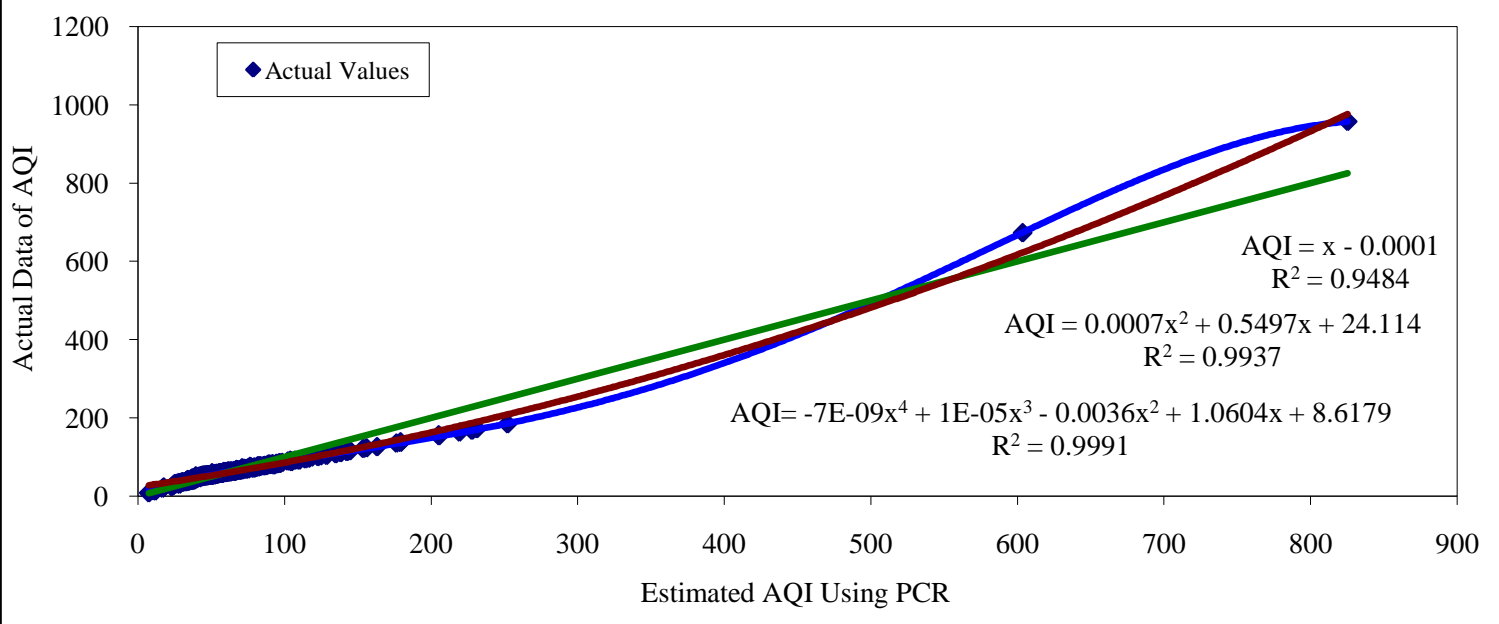

Fig. 4: Estimated AQI Using PCR of Varimax Method Versus Actual Data 
DOI: https://dx.doi.org/10.26808/rs.ca.i8v1.01

International Journal of Computer Application (2250-1797)

Issue 8 Volume 1, January- February 2018

\section{CONCLUSION}

In this work, PCA and ANN are merged (PC-ANN) to predict AQI based on 10 historical air quality and meteorological parameters. Four different approaches are used. The first approach is based on original data (10 original parameters), as well as, the other approaches are based on rotated PCs using Varimax, Equamax and Quartimax in order to obtain the inherent variables to feed-forward ANN and MLR inputs. Using four $\mathrm{PCs}$, significant loadings are observed for $\mathrm{CO}, \mathrm{NO}, \mathrm{NO}_{2}, \mathrm{NO}_{\mathrm{X}}, \mathrm{PM}_{10}, \mathrm{SO}_{2}$, Wind Direction (WD), Wind Speed (WS), Ambient Temperature (AT) and Sigma Theta (SGT). ANN and MLR models based on original raw data, as well as PC-ANN and PCR models based on four PC scores, are compared to obtain the accurate AQI prediction.

The results show that the feed-forward ANN model, using ten original parameters as inputs, gives a high value of $\mathrm{R}^{2}$ and low values of error rates that contrast to results from MLR model (Method 1). However, Method 2 (PC-ANN using Varimax method) gives better prediction of the results as compared to Method 1 in term of $\mathrm{R}^{2}$ value and error rate values. Furthermore, the relation between Varimax PC-ANN prediction results and actual values are almost matching. The prediction performance of the Methods 3 and 4 (PC-ANN using Equamax and Quartimax methods, respectively) gives lower effectiveness than Methods 1 and 2, but these methods can predict the AQI within acceptable accuracy when compared with MLR and PCR models. The results proved that the merge of Varimax rotated PC scores with ANN model is more efficient and precise in the estimation of AQI results. Moreover, using ANN or PC-ANN model gives more accurate estimation results than MLR or PCR. Whereas it also proves that, the ANN and PC-ANN models are very helpful tools in making decision and solving problem for better atmospheric management of the local environment.

\section{REFERENCES}

[1] How air pollution can cause cancer, Cancer Research UK, http://www.cancerresearchuk.org/about-cancer/causes-of-cancer/air-pollution-radon-gas-and-cancer/howair-pollution-can-cause-cancer

[2] Kamal Md. M., Jailani R., \& Shauri R. L. A.; Prediction of Ambient Air Quality Based on Neural Network Technique; In SCOReD 2006 -Proceedings of 2006 4th Student Conference on Research and Development "Towards Enhancing Research Excellence in the Region”, pp. 115-119, 2006.

[3] Bishoi B., Prakash A. and Jain V. K.; A Comparative Study of Air Quality Index Based on Factor Analysis and US-EPA Methods for an Urban Environment; Aerosol and Air Quality Research, Vol. 9, No. 1, pp. 1-17, 2009.

[4] Rao V. L.; An Estimation of Air Quality Index of a Coastal Station A case study; International Journal of Microbiology and Applied Science, ISSN: 2319 -7706, Vol. 3, No. 6, pp.759-763, 2014.

[5] Environmental Protection Agency (EPA); Air Quality Index Reporting Final Rule; Part III, Federal Register, Vol. 64, No. 149, Rules and Regulations, 1999.

[6] Radiation Monitoring Network in Egypt.

[7] Yamartino, R. J.; A Comparison of Several "Single-Pass" Estimators of the Standard Deviation of Wind Direction; Journal of Climate and Applied Meteorology. Vol. 23, No. 9, pp. 1362-1366, 1984.

[8] Paynter D.; Calculation and Re-Calculation of 60-Minute Sigma Theta and Stability-2003;

hps.ne.uiuc.edu/numug/archive/2003/presentations/paynter.pdf

[9] Isiyaka H. A. and Azid A.; Air Quality Pattern Assessment in Malaysia Using Multivariate Techniques; Malaysian Journal of Analytical Sciences, Vol. 19, No. 5, pp. 966 -978, 2015.

[10] Azid A., Juahir H., Latif M. T., Zain Sh. M. and Osman M. R.; Feed-Forward Artificial Neural Network Model for Air Pollutant Index Prediction in the Southern Region of Peninsular Malaysia; Journal of Environmental Protection, No. 4, pp.1-10, 2013. 
DOI: https://dx.doi.org/10.26808/rs.ca.i8v1.01

[11] Technical Assistance Document for the Reporting of Daily Air Quality - the Air Quality Index (AQI), U.S. Environmental Protection Agency Office of Air Quality Planning and Standards Research Triangle Park, North Carolina 27711, 2016.

https://www3.epa.gov/airnow/aqi-technical-assistance-document-may2016.pdf

[12] Ul-Saufie A. Z., Yahya A. Sh., Ramli N. A. and Abdul Hamid H.; Comparison Between Multiple Linear Regression And Feed forward Back propagation Neural Network Models For Predicting PM10 Concentration Level Based On Gaseous And Meteorological Parameters; International Journal of Applied Science and Technology, Vol. 1, No.4, 2011.

[13] Dominick D., Juahir H., Latif M. T., Zain Sh. M. and Aris A. Z.; Spatial assessment of air quality patterns in Malaysia using multivariate analysis; Atmospheric Environment, Vol. 60, pp. 172-181, 2012.

[14] SPSS Statistics Base 17.0 User's Guide Manual, https://www.jou.ufl.edu/assets/researchlab/SPSS-Statistcs-Base-Users-Guide-17.0.pdf

[15] Khan R. A., Zain Sh. M., Juahir H., Yusoff M. K. and Tg Hanidza T. I.; Using Principal Component Scores and Artificial Neural Networks in Predicting Water Quality Index; Chemometrics in Practical Applications Edited by Dr. Kurt Varmuza, Chapter 12, 2012.

[16] Azid A. et al. ; Identification Source of Variation on Regional Impact of Air Quality Pattern Using Chemometric; Aerosol and Air Quality Research, Vol.15, pp. 1545-1558, 2015.

[17] Michy Alice,July 20, 2016,Performing Principal Components Regression (PCR) in R, accessed 29 Mars 2017.

https://www.r-bloggers.com/performing-principal-components-regression-pcr-in-r/

[18] Zekić-Sušac M., Šarlija N. and Pfeifer S.; combining PCA Analysis and Artificial Neural Networks in Modelling Entrepreneurial Intentions of Students, Croatian Operational Research Review (CRORR), Vol. 4, pp. 306-317, 2013.

[19] MINITAB User's Guide 2, Data Analysis and Quality Tools, Release 13, USA, Ch. 7, 2000. 